\title{
Central Nervous System Mesenchymal Chondrosarcoma
}

National Cancer Institute

\section{Source}

National Cancer Institute. Central Nervous System Mesenchymal Chondrosarcoma. NCI

Thesaurus. Code C129534.

A mesenchymal chondrosarcoma that arises from the central nervous system. 